\title{
Genome-wide analyses of rice root development QTLs and development of an online resource, Rootbrowse
}

\author{
Pala Suryapriya, Allada Snehalatha, Ulaganathan Kayalvili, Radha Krishna, Sukpal singh and Kandasamy \\ Ulaganathan* \\ Centre for Plant Molecular Biology, Osmania University, Hyderabad 500 007, India; \\ K. Ulaganathan* - Email: kulaganathan123@gmail.com; * Corresponding author
}

received December 11, 2008; revised December 19, 2008; accepted December 29, 2008; published January 12, 2009

\begin{abstract}
:
Genetic control of root development in rice is complex and the underlying mechanisms (constitutive and adaptive) are poorly understood. Lowland and upland varieties of indica and japonica rice with contrasting root development characteristics have been crossed, mapping populations developed and a number of QTLs in different chromosomes were identified. As these studies have used different sets of markers and many of the QTLs identified are long, it is difficult to exploit the varietal difference for improved root traits by marker assisted selection and for identification of concerned alleles. Intensive data mining of literature resulted in the identification 861 root development QTLs and associated microsatellite markers located on different chromosomes. The QTL and marker data generated and the genome sequence of rice were used for construction of a relational database, Rootbrowse, using MySQL relational database management system and Bio::DB::GFF schema. The data is viewed using GBrowse visualization tool. It graphically displays a section of the genome and all features annotated on it including the QTLs. The QTLs can be displayed along with SSR markers, protein coding genes and/or known root development genes for prediction of probable candidate genes.
\end{abstract}

Keywords: root development; rice; QTLs; candidate genes; auxin metabolism; gbrowse

Abbreviations: QTLs - quantitative trait loci; SSR - simple sequence repeats

Availability: Rootbrowse is freely available at http://www.ricebrowse.org.

\section{Background:}

Rice with its poor water use efficiency needs about 5000 liters of water to produce $1 \mathrm{~kg}$ of rice and availability of water is the major limiting factor in rice production in irrigated and rain-fed ecosystems due to shrinking ground water resources and frequent occurrence of drought. Drought is a source of huge yield loss. Rice plant architecture [1] has been found to affect various aspects of yield [2] and it has been shown that the plants respond by architectural changes to various stress factors. Roots are an important part of the plant architecture that is involved in foraging for the available water and nutrients in the rhizosphere [3]. A deep and thick root system is generally considered as a more favorable element allowing the crop to maintain its water status even under water stress [4].

Upland rice varieties, grown with no irrigation, usually have larger and deeper roots as compared to lowland rice varieties [5, 6]. Deep root development, which is important for the drought resistance in rice (Oryza sativa L.), is a complex trait combining various root morphologies and show considerable genotypic variation $[7,8]$. A deep and thick root system has been thought to be advantageous for improved drought tolerance in rain fed lowland ecosystem [9]. Both in upland and lowland rice, the root traits can be broadly divided into constitutive root traits and adaptive root traits. Constitutive root traits control the level of root development under normal growth conditions. Adaptive root traits control the level of root development under stress conditions i.e. as an induced response in the root architecture. Rice varieties show diversity in adaptive as well as constitutive traits for root development $[\mathbf{1 0}, \mathbf{1 1}]$.

\section{Methodology: \\ Database construction}

Intensive search made for the scientific papers on root development of rice resulted in identification of seventeen studies [11-26]. These studies were based on mapping populations (RILs, DHLs, BILs or NILs) produced using Indica lowland varieties (IR64, IR1552, IR62266, IR58821, IR52561, Co39 and Zhenshan97), Indica upland varieties (Bala and Kalinga III), Japonica lowland varieties (Akihikari and Yuefu) and Japonica upland varieties (CT9993, IAC165, IRAT109, and Azucena) [27]. These were used for data mining which resulted in the identification of a total of 861 root development QTLs and associated markers located on different chromosomes. Chromosome 1 had 135 QTLs, chromosome 7 had 88 QTLs, chromosome 2 had 87 QTLs, chromosome 9 had 84 QTLs and chromosome 4 had 82 QTLs. Chromosome 6 and Chromosome 5 had 78 and 77 QTLs respectively. Chromosome 8 and chromosome 11 had 56 QTLs each. Chromosome 3 had 51 QTLs. Chromosome 12 had 39 QTLs. The least number of QTLs on the basis of 


\section{Bioinformation}

chromosomal distribution was found to be on Chromosome 10 which had 28 QTLs located on it. Using the marker sequences all these 861 QTLs were integrated with the sequence of O.sativa ssp.japonica var. Nipponbare. Genome-wide intensive search for simple sequence repeats was made and all simple sequence repeats were included in the database. The QTL and marker data generated and the genome sequence of rice [28] were used for construction of a relational database using MySQL relational database management system and Bio::DB::GFF schema. Further we carried out genome-wide analysis for root development related genes using heterologous genes identified in other plants species. These genes are also part of the database and can be viewed in combination with the QTLs.

\section{QTL Visualization and Candidate gene prediction}

The data from the database is visualized using the GBrowse visualization tool [29] which graphically displays a section of the genome and all features annotated on it including the QTLs (Figure 1). The user can zoom in and out and scroll through the genome and click on features to obtain more detailed information. Users can specify a genome segment for displaying, e.g. chr1:1000..9000, or query the database by entering a keyword including wild card characters e.g. root*. This query will return a list of matches to the search term. The QTLs can be searched using the trait name (e.g. Deep root), variety (e.g. Azucena) and author name (e.g. Yadav). The QTLs can be displayed along with known polymorphic SSR markers, predicted SSR markers, protein coding genes and/or known root development genes (including genes associated with auxin metabolism). Rootbrowse is a unique integrated resource where analyses of QTLs can be carried out in the genomic context for prediction of probable candidate genes and for identification of markers flanking the predicted genes. This resource will also aid marker assisted selection for root development by in silico fine mapping that can be verified in the population. The Rootbrowse database can be accessed at the address: http://www.ricebrowse.org.

\section{Future development:}

The database will be expanded by including stress QTLs and sequence variation in protein coding genes, miRNAs and their probable binding sites. It is also proposed to include microarray data of genes expressed in root tissues.

\section{Acknowledgment:}

Pala Suryapriya is a recipient of senior research fellowship awarded by University Grants Commission (UGC), New Delhi, INDIA, A.Snehalatha and A. R. Krishna are recipients of senior research fellowships awarded by the Council of Scientific and Industrial Research (CSIR), New Delhi, INDIA. We thank the Director, Centre for Plant Molecular Biology, and Osmania University for infrastructural support.

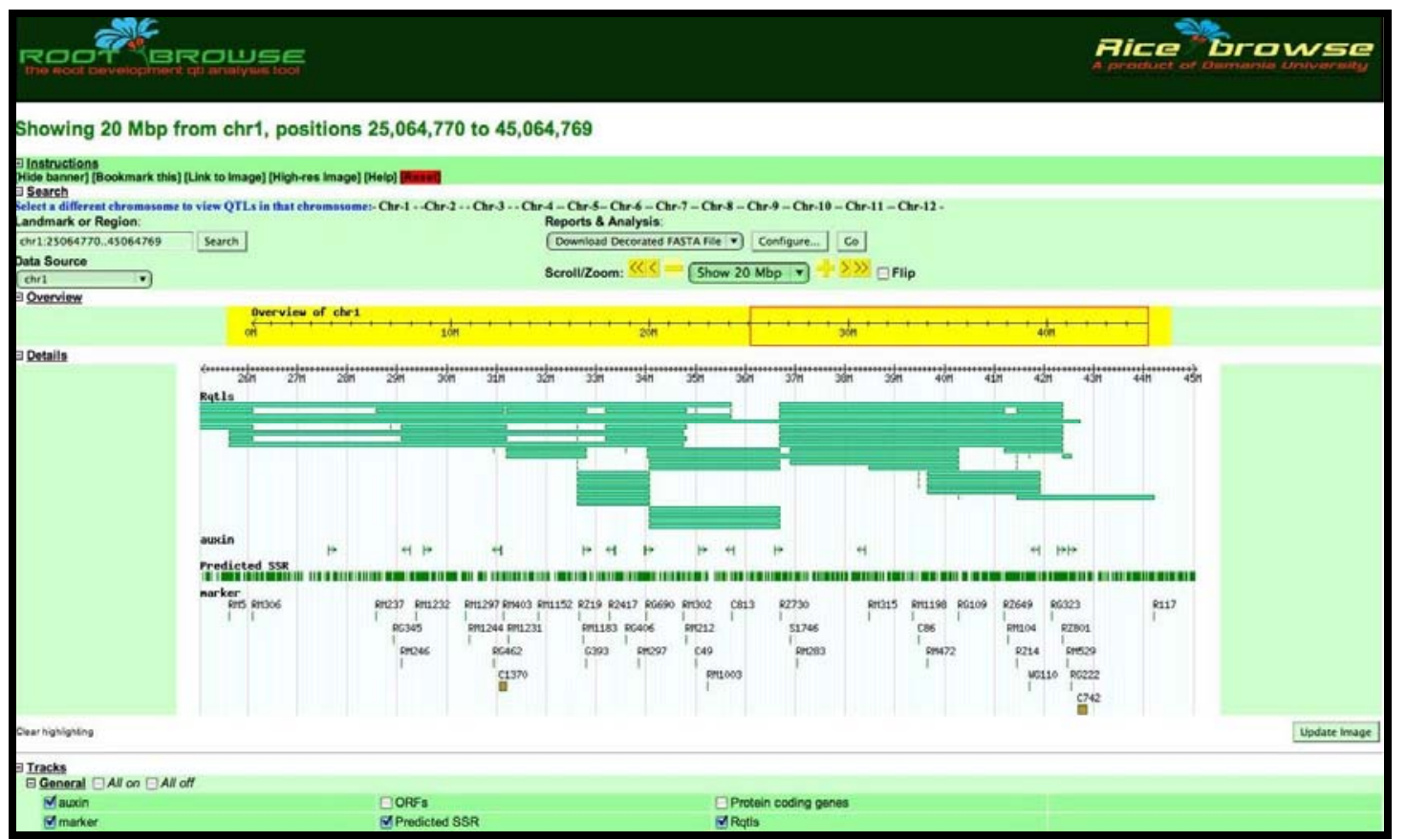

Figure 1: Snapshot of Gbrowse view of QTLs, SSR markers and genes associated with auxin metabolism in Chromosome 1 of rice.

\section{References:}

[01] J. I. Itoh et al., Pl cell physiol., 46: 23 (2005)

[02] D.Barthelemy et al., Ann Bot., 99: 375 (2007)

[03] J. E. Malamy, Pl Cell and Environ., 28: 67 (2005)

[04] H. T. Nguyen et al., Crop Sci., 37: 426 (1997)

[05] H.Araki et al., Plant andl Soil, 270: 147 (2005)

[06] J. M. Lilley et al., Field Crop Res., 37: 205 (1994)
[07] Y. Kato et al., Plant and Soil, 287: 117 (2006)

[08] B. Mambani et al., Plant and Soil, 73: 59 (1983)

[09] S. Fukai et al., Field Crop Res., 40: 67 (1995)

[10] Azhiri-Sigari et al., Plant Prod Sci., 3: 173 (2000)

[11] A. Kamoshita et al., Crop Science, 42: 255 (2002)

[12] R.Yadav et al., Theor Appl Genet., 94: 619 (1997) 


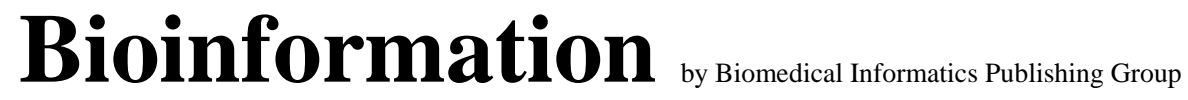

open access

\section{www.bioinformation.net}

Database

[13] A. H. Price et al., Theor Appl Genet., 100: 49 (2000)

[14] M. L. Ali et al., Theor Appl Genet., 101: 756 (2000)

[15] L. Shen et al., Theor Appl Genet., 103: 75 (2001)

[16] J. Zhang et al., Theor Appl Genet., 103: 19 (2001)

[17] W. P. Zhang et al., Theor Appl Genet., 103: 118 (2001)

[18] A. H. Price et al., J Exp Bot., 53: 989 (2002)

[19] B. Courtois et al., Euphytica, 134: 335 (2003)

[20] C. S. Zheng et al., Theor Appl Genet., 107: 1505 (2003)

[21] Z. Li et al., Theor Appl Genet., 110: 1244 (2005)
[22] H. Horii and K. Nemoto, Plant Breeding 125: 198 (2006)

[23] K. Macmillan et al., Theor Appl Genet., 113: 977 (2006a)

[24] K. Macmillan et al., Theor Appl Genet., 113: 953 (2006b)

[25] K. A. Steele et al., Theor Appl Genet., 112: 583 (2006)

[26] L. Liu et al., Euphytica, 164: 729 (2008)

[27] K. Ulaganathan et al., Mol Pl Breeding, 5: 213 (2007)

[28] T. Tanaka et al., Nucleic Acids Res., 36: D1028(2008)

[29] L. D. Stein et al., Genome Res., 10: 1599 (2002)

Edited by $P$. Kangueane

Citation: Suryapriya et al., Bioinformation 3(6): 279-281 (2009)

License statement: This is an open-access article, which permits unrestricted use, distribution, and reproduction in any medium, for non-commercial purposes, provided the original author and source are credited. 